\title{
Analysis of dietary habits and health status in a court of young athletes in Northern Italy practicing Athletic disciplines
}

\section{Abstract}

Introduction: A balanced diet is a fundamental component of athletes' health, training and performance. WHO dietary guidelines highlight the importance of maintaining a normal BMI, limiting the intake of sugars (especially sweetened foods and beverages), eating at least 5 portions of fruits and vegetables per day, drink lots of clean water (at least 2 L per day for children older than 13 and for adults). HBSC-Italy study reveals that children have frequently bad eating habits, such as consuming sweetened drinks and not enough fruits and vegetables. Indeed, $21.3 \%$ of Italian children are overweight and $9.3 \%$ are obese. The purpose of this study is to evaluate nutritional and health status of a population of Northern Italy children and adolescents practicing Athletics, investigating for both endurance and not endurance disciplines. Data obtained are a starting point for focused food education interventions aimed at improving health and sport performance.

Materials and Methods: A cohort of 125 athletes (12-25 years, 42.4\% male, $57.6 \%$ female). Two groups were created: children (12-17 years) and adults (18-25 years). Weight, height, waist circumference, triceps and subscapularis skinfolds were measured in order to calculate BMI, Waist-to-Height ratio (WtHr), body adiposity and muscle mass. At baseline and after 1 year (follow-up) from a nutritional educational intervention, the anthropometric measures and dietary habits were tested with a validated dietary screening survey, considering food quality, portions and frequencies of consumption, were taken.

Results: At Baseline, more than $85 \%$ of athletes were found to be normal weight with WHtR $<0.5$. All the athletes showed body adiposity in the normal range. Regarding food habits, most athletes do not take the recommended daily fruit and vegetable servings follow Italian trend. Only about $20 \%$ of children athletes assumes the water daily intake. On the contrary, about $50 \%$ of athletes in every group drink sweetened beverages every day. The data collection after the personalized food education meetings will be collected in May 2019.

Discussion: The athletes of cohort shows a good health status but nutritional habits present critical issue. For this reasons, there is a need of nutritional education for improving healthy eating habits in young athletes in order to impact positively on future health status and on sport performance.

\section{Conflict of Interest}

There is no conflict of interest 